\title{
A comparison of the impact of central ECRH and central ICRH on the tungsten behaviour in ASDEX Upgrade H-mode plasmas
}

\author{
C. Angioni ${ }^{1}$, M. Sertoli ${ }^{1}$, R. Bilato ${ }^{1}$, V. Bobkov ${ }^{1}$, A. Loarte $^{2}$, R. \\ Ochoukov $^{1}$, T. Odstrcil ${ }^{1}$, T. Pütterich ${ }^{1}$, J. Stober $^{1}$ and the \\ ASDEX Upgrade Team \\ 1 Max-Planck Institut für Plasmaphysik, Garching, Germany \\ 2 ITER Organization, Route de Vinon-sur-Verdon, CS 90 046, 13067 St Paul Lez \\ Durance, France
}

\begin{abstract}
A comparison of the impact of additional central electron cyclotron resonance heating (ECRH) and ion cyclotron resonance heating (ICRH) on the behaviour of the tungsten (W) density in the core of H-mode plasmas heated with neutral beam injection (NBI) is performed in ASDEX Upgrade. Both localized and broad profiles of the power density of the ECRH have been obtained, where broad profiles reproduce the profile shape of the ICRH power density, which is applied with a hydrogen minority heating scheme. In contrast to ECRH, which produces direct electron heating only, ICRH eventually heats both electrons and ions in almost equal fractions. It is found that both additional RF heating systems reduce the peaking of the $\mathrm{W}$ density profile with increasing central RF heating power. Approximately the same values of $\mathrm{W}$ density peaking are obtained when the same values of electron heating are produced by the two RF heating systems, which implies that less total heating power is required with ECRH than with ICRH to reduce the $\mathrm{W}$ density peaking. A related modelling activity shows that an important ingredient to explain the experimentally observed trend is the variation of the turbulent $\mathrm{W}$ diffusion as a function of the electron to ion heat flux ratio. Additional effects are connected with the more favorable $\mathrm{W}$ neoclassical transport convection in the presence of ICRH, produced by the combination of stronger central ion temperature gradients and the impact of the $\mathrm{H}$ minority on the $\mathrm{W}$ poloidal density asymmetry.
\end{abstract}

PACS numbers: 52.55.Fa, 52.25.Fi, 52.25.Vy, 52.65.Tt 


\section{Introduction}

Heavy impurities are expected to play a critical role in a fusion reactor plasma, not only because tungsten ( $\mathrm{W}, \mathrm{Z}=74, \mathrm{~A}=184$ ) is currently considered as a first wall material, but also because highly charged impurities are planned to be seeded in order to radiate part of the heating power in the plasma periphery and reduce the power loads directly reaching the walls. Thereby, robust methods by which the impurity concentration and the shape of the impurity density profile can be kept under control have to be identified. These are determined by the combination of the effects of the peripheral sources and the transport from the scrape-off-layer to the plasma core. Central radio frequency (RF) heating has been identified as a reliable method to limit the central concentration of heavy impurities [1-9]. Since the behaviour and the profile shapes of heavy impurities are determined by both turbulent [10-23] and neoclassical transport [25-30], and are also affected by the presence of magneto-hydrodynamic (MHD) instabilities [6,31-35], the physics behind the reduction of the central peaking of the $\mathrm{W}$ density produced by central RF heating can be expected to involve a certain level of complexity, in which multiple effects are combined. Central RF heating not only modifies the temperature, density and rotation profiles of the main plasma, but can also directly modify the impurity transport. Central accumulation (that is, a $\mathrm{W}$ density profile centrally more peaked than the electron density profile) is produced by the dominance of neoclassical transport in the unfavourable conditions of inward neoclassical convection (pinch) [1,2,33,36-44]. Turbulent transport is not predicted to lead to strong central accumulation (e.g. [13]) and thereby an increase of turbulent impurity transport can offset the neoclassical pinch. Finally, central RF heating can modify the characteristics of MHD instabilities, particularly at the $\mathrm{q}=1$ surface, with impact on the impurity behavior $[6,31,32,34]$.

Here we report the results obtained in an experiment at ASDEX Upgrade (AUG) in which plasma discharges have been performed in order to compare the impact of central electron cyclotron resonance heating $(\mathrm{ECRH})$ and ion cyclotron resonance heating $(\mathrm{ICRH})$, where the latter is applied with a $\mathrm{H}$-minority heating scheme. Decreasing steps of RF heating power have been added to a background of neutral beam injection (NBI) heating power during the current flat-top phase of the plasma discharges, with the plasma in the $\mathrm{H}$-mode confinement regime. Both localized and broad power deposition profiles of the ECRH have been produced, where the broad profile reproduces the power deposition profile shape produced by central ICRH. The experimental results confirm the beneficial role of central RF heating in reducing the central peaking of the $\mathrm{W}$ density, consistent with previous studies. In addition they allow a direct comparison of the two $\mathrm{RF}$ heating methods and reveal that comparable effects on the $\mathrm{W}$ density are produced when similar amounts of additional electron heating are delivered to the plasma. This observation implies that, at least in these conditions with a background of large NBI heating, ECRH is more efficient in reducing the peaking of the $\mathrm{W}$ density, since it delivers all of the power to the electrons, whereas, with the heating scheme applied in these experiments, ICRH also has a fraction of the power which heats the ions. The critical role of electron heating in flattening the central density profile of heavy impurities 
revealed by these experiments appears to be consistent with previous results at JET [4], where nickel $(\mathrm{Ni})$ density profiles were observed to be flat with central ICRH in mode conversion, producing dominant electron heating, and peaked with central ICRH in $\mathrm{He}^{3}$ minority heating scheme, producing dominant ion heating, although also with different power deposition profiles. Moreover, higher values of the central diffusion coefficient of silicon ( $\mathrm{Si}$ ) were measured in ASDEX Upgrade with central ECRH in comparison to central ICRH [2], although also in this case the power deposition profiles of ECRH were more centrally localized than those of ICRH.

In order to identify the transport mechanisms which can explain the new observations reported in this paper, power density profiles of ECRH and ICRH have been computed with the codes TORBEAM [45] and TORIC-SSFPQL [46], and complete power balance calculations have been obtained with TRANSP [47]. In addition, experimentally reconstructed profiles of the $\mathrm{W}$ density are compared with the predictions obtained by combining the results of codes which separately compute neoclassical and turbulent transport, drift-kinetic NEO [48-50] and gyro-kinetic GKW [51,52] respectively. The modelling activity is particularly devoted to identify reasons which can at least partly explain the observed positive role of electron heating produced by the RF heating systems. We also note that these codes have been run assuming axisymmetric geometry, that is in the absence of any perturbation of the confining magnetic field produced by MHD modes.

In Section 2 the experiment is described and the experimental results are presented. In section 3 the observations are modelled in order to shed light on the relative role of the transport mechanisms governing the W density behaviour. Finally in Section 4 conclusions are drawn.

\section{Experimental investigation of the $W$ response to central ECRH and ICRH}

In order to compare the impact of central ECRH and central ICRH on W transport in otherwise similar conditions, plasma discharges of $1 \mathrm{MA}$ and magnetic field around $2.5 \mathrm{~T}$ $\left(q_{95} \simeq 4\right)$ have been produced in ASDEX Upgrade at the line averaged electron density of $n_{e}=7.210^{19} \mathrm{~m}^{-3}$, with the current flat top phase in the H-mode confinement regime, heated by $7.5 \mathrm{MW}$ of constant NBI heating power and by decreasing power steps of central ECRH (from 1.9 MW to 0.2 MW) and ICRH (from 3.6 MW to 0.2 MW). ICRH is applied with a $\mathrm{H}$-minority heating scheme, with a $\mathrm{H}$ concentration around $5 \%$, with both the 2-straps antenna with boron coated limiters and the 3-straps antenna with tungsten coated limiters [53]. 3 gyrotrons at $140 \mathrm{GHz}$ [54] have been used for ECRH. Intermediate power steps of ECRH are obtained by modulation of the power at the high frequency of $150 \mathrm{~Hz}$ and with $50 \%$ duty cycle for each gyrotron. Time traces of two discharges with decreasing power steps of ICRH and ECRH are presented in Fig. 1 and Fig. 2 respectively. With decreasing RF power (Figs. $1 \mathrm{~b}$ and $2 \mathrm{~b}$ ), the $\mathrm{W}$ concentration at the center (Figs. 1c and 2c) (obtained from a fit of the spectrum around $5 \mathrm{~nm}$ measured on a single central line-of-sight by the grazing incidence spectrometer (GIW)) 


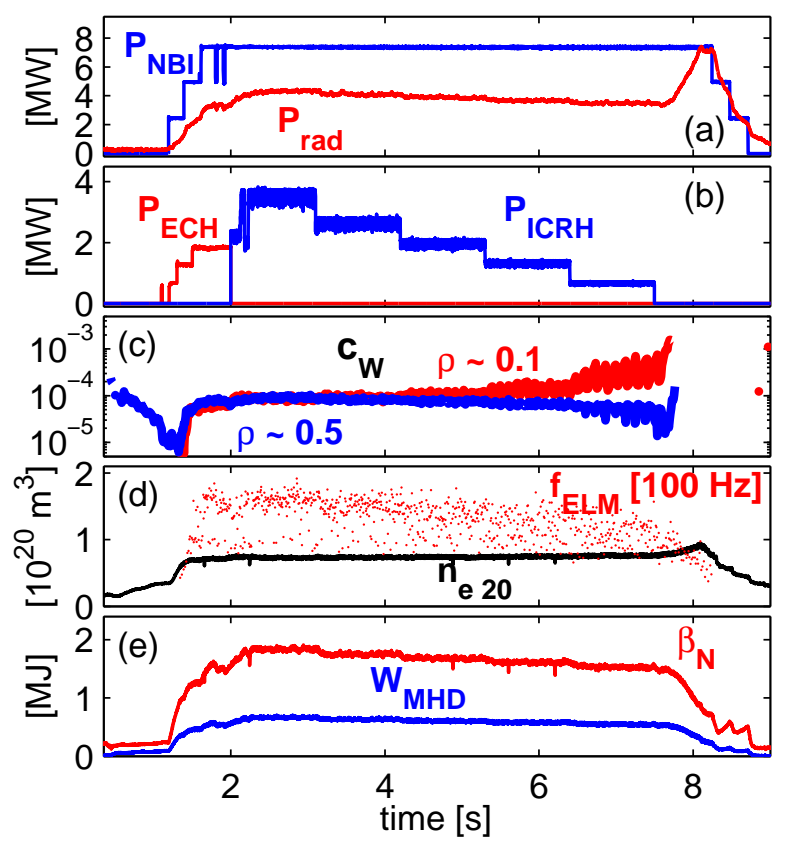

Figure 1. Time traces of AUG shot $\sharp 32404$ with decreasing steps of ICRH power, total NBI and radiated powers (a), total ECH and ICRH powers (b), W concentration $c_{W}=n_{W} / n_{e}$ at $\rho \simeq 0.1$ and $\rho \simeq 0.5$ (c), ELM frequency and line averaged density (d), normalized $\beta$ and total stored energy (e).

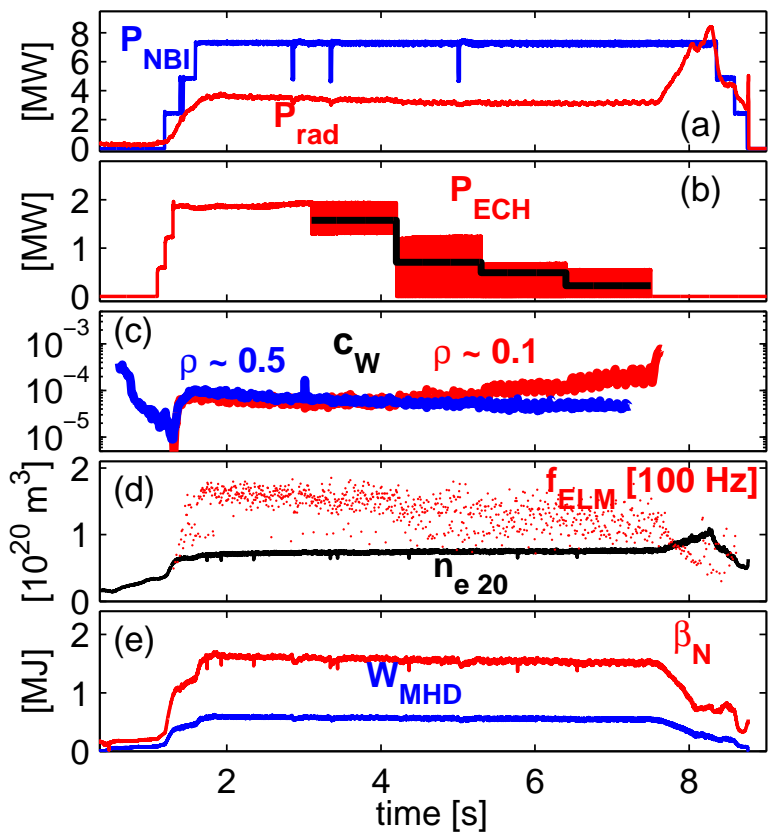

Figure 2. Time traces of AUG shot $\sharp 32408$ with decreasing steps of ECRH power, total NBI and radiated powers (a), total ECH and ICRH powers (b), W concentration $c_{W}=n_{W} / n_{e}$ at $\rho \simeq 0.1$ and $\rho \simeq 0.5$ (c), ELM frequency and line averaged density (d), normalized $\beta$ and total stored energy (e). 
progressively increases, eventually leading to central accumulation, as also shown by the corresponding fast increase of the total radiated power (Figs. 1a and 2a). The level of gas puff, by which the ELM frequency can be regulated and the $\mathrm{W}$ concentration at the pedestal top can be limited, is kept to low values in these discharges, and sudden uncontrolled W accumulation is observed in the presence of NBI heating only (usual in ASDEX Upgrade H-modes at high plasma currents [3]).

An interesting observation is that, during the power steps with relatively low levels of RF heating, the $\mathrm{W}$ behaviour can reach stationary conditions with a $\mathrm{W}$ density profile which is significantly more peaked than the electron density profile. Thereby, strictly speaking, W accumulation is observed, but this does not lead to an uncontrolled process of increasing accumulation, which instead suddenly takes place in conditions of NBI only heating, as shown for instance in Figs. 1 and 2. This property is illustrated in Fig. 3 where the time evolutions of the flux-surface-averaged $\mathrm{W}$ density profiles (reconstructed by a SXR W density diagnostic [55]) are plotted as a function of normalized minor radius $r / a$ and time during high $(\mathrm{a}, \mathrm{d})$ and low $(\mathrm{b}, \mathrm{e})$ additional RF power phases of the two discharges with additional ICRH $(\mathrm{a}, \mathrm{b})$ and ECRH $(\mathrm{d}, \mathrm{f})$, whose time traces were already presented in Figs. 1 and 2 respectively. In Fig. 3 (a,b,d,e), the oscillations of the W density are produced by sawteeth. In Fig. 3(c,e), the time averaged W density profiles are compared to the corresponding electron density profiles. The profiles are normalized to their values at $r / a=0.4$ (that is, all of the normalized profiles cross 1 at $r / a=0.4$ ). Fig. 3(c,e) demonstrate that in phases with high RF power no accumulation takes place, whereas in phases with low RF power, stationary conditions are achieved which exhibit significant $\mathrm{W}$ accumulation.

Since in AUG the ICRH power deposition profiles are relatively broad as compared to the possibilities of the ECRH power, discharges with decreasing power steps of ECRH have been applied with 3 gyrotrons all converging to a deposition close to the magnetic axis $\left(\rho_{\text {dep }} \simeq 0.1\right)$ as well as with 3 gyrotrons aiming at different radial locations in order to approximatively reproduce the profile of the deposited power from ICRH (as obtained by TORIC-SSFPQL [46] simulations applied to a previous similar plasma). A comparison of the profiles of the deposited power with localized ECRH, with broad ECRH, (computed by TORBEAM [45]) as well as with ICRH (computed with TORICSSFPQL [46]) are presented in Fig. 4. We also observe that, in comparison with the RF power profiles, the power density and heat flux profiles produced by NBI (computed with TRANSP [47]) are much broader than the corresponding profiles produced by the RF heating systems (4 a,b). However, considering the actual levels of the heat fluxes in MW (4 c), in the central region the NBI heat flux is comparable to that of broad ECRH at the maximum power step $(1.9 \mathrm{MW})$, whereas the ICRH heat flux profile at the maximum power step of 3.4 MW is closer to that obtained with localized ECRH at 1.9 MW. In these discharges, in almost the totality of the heating phases, sawteeth are present, with sawtooth periods of the order of $100 \mathrm{~ms}$ and sawtooth inversion radius usually located between $r / a=0.3$ and $r / a=0.35$. In the analysis we consider profiles which are averaged over time windows of about $500 \mathrm{~ms}$, which contain multiple sawtooth cycles and which provide averaged profiles over the sawtooth oscillations. We observe 


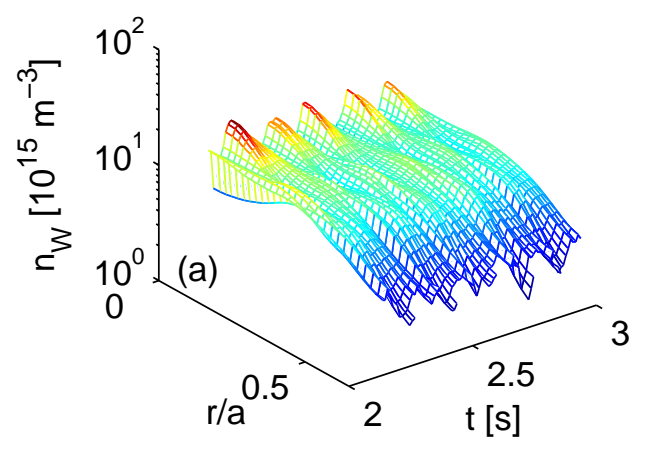

AUG \#32404 from 6.6 s to $7.5 \mathrm{~s}$
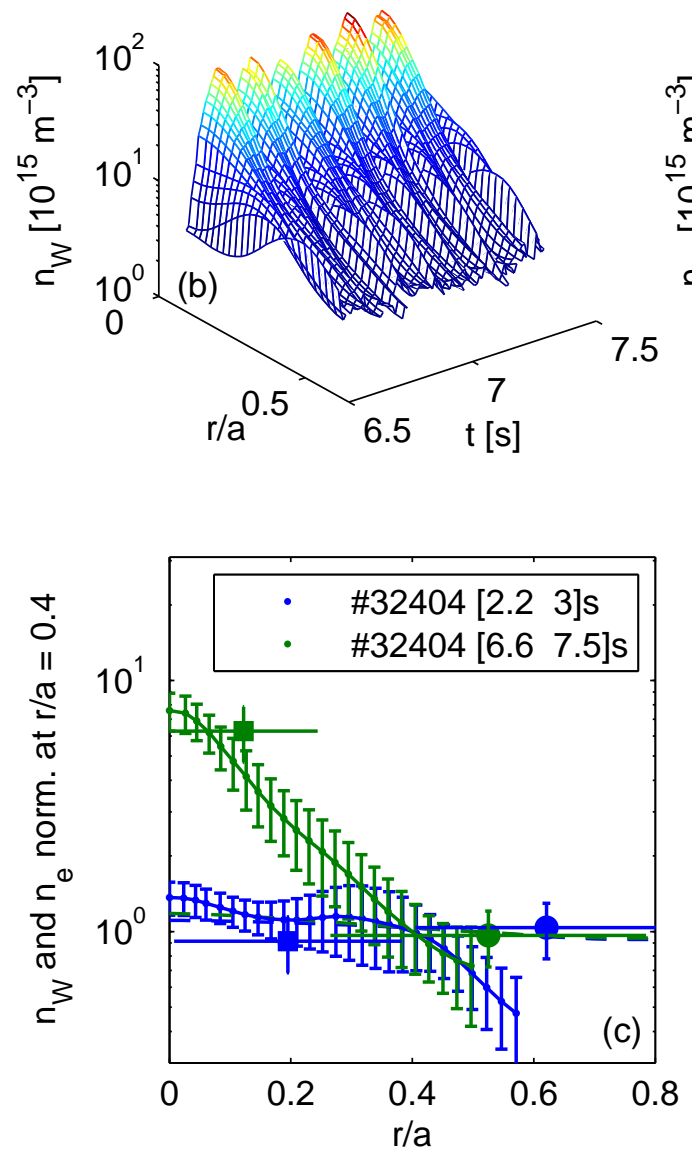

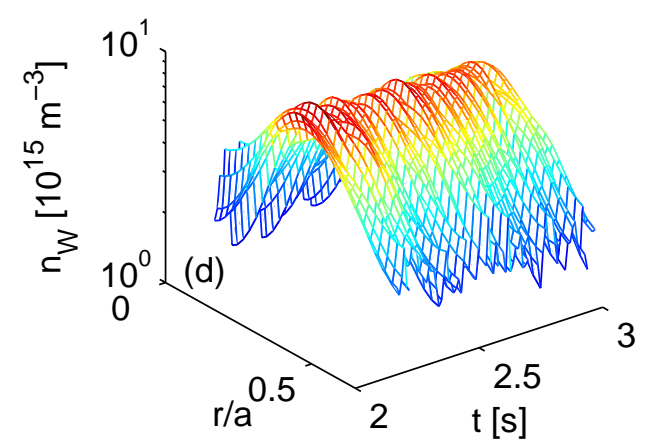

AUG \#32408 from 6.6 s to $7.5 \mathrm{~s}$
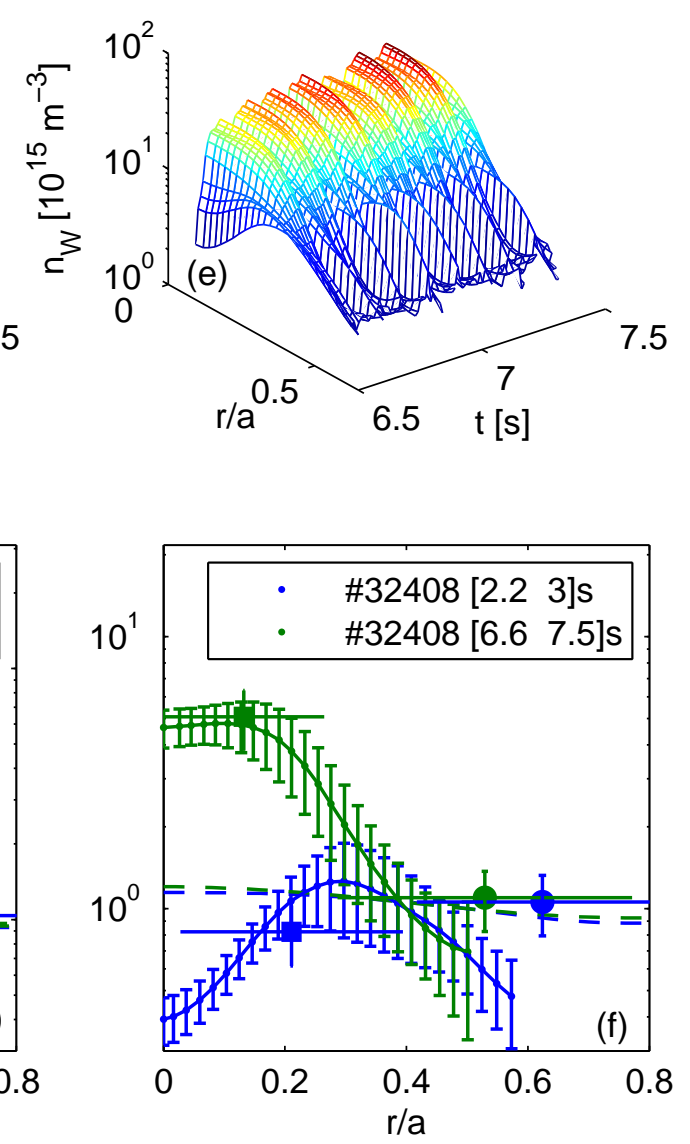

Figure 3. Time evolution of the SXR-evaluated W density profiles with ICRH (a-c) and ECRH (d-f) at high (time window from 2.2s to 3.0) (a,b) and low (time window from $6.6 \mathrm{~s}$ to $7.5 \mathrm{~s}$ ) (c,d) RF power density, and (e,f) corresponding time averaged W density profiles (solid) compared to the corresponding time averaged electron density profiles (dashed). all normalized to their values at $\mathrm{r} / \mathrm{a}=0.4$. In $(\mathrm{e}, \mathrm{f})$, full squares and full circles are the $\mathrm{W}$ corresponding density measurements of the GIW spectrometer (core and peripheral respectively) multiplied by the electron densities at the middle of the radial intervals of measurement (plotted with horizontal bars), and normalized in the same way as the SXR-evaluated W density profiles. 


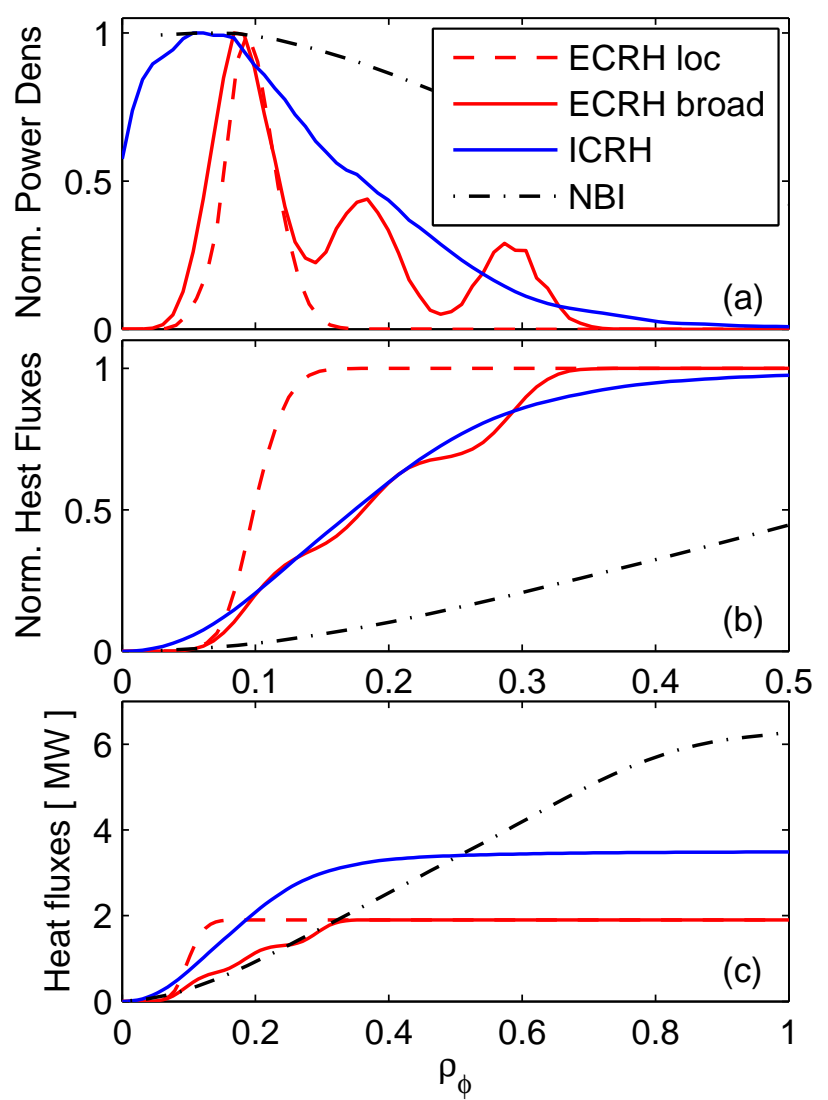

Figure 4. Power density (a) and integrated power (b,c) profiles with localized and broad ECRH (TORBEAM) [45] and with ICRH (TORIC-SSFPQL) [46] as well as NBI (TRANSP) normalized to the maximum power density in (a) and normalized to the total injected power in (b), in actual MW in (c). In (c) the phases with maximum heating power of ECRH (1.9 MW) and ICRH (3.4 MW) are considered. Different $\mathrm{x}$-axis are used in $(\mathrm{a}, \mathrm{b})$ and in $(\mathrm{c})$.

that when the sawtooth crashes produce a significant modification of the plasma kinetic profiles in the central region, the recovery time is usually short as compared to the sawtooth period. Therefore, time averaged profiles are not very different from the profiles measured just before the sawtooth crashes. An analysis based on pre-crash plasma conditions can be expected to give results which are consistent with those which are presented here, and which have been obtained based on plasma conditions time averaged over multiple sawteeth.

In AUG, ICRH is accompanied by an additional W source as compared to ECRH, which has been recently minimized by using the new 3-strap ICRF antennas [53]. Discharges with ECRH power scans have been also performed with additional W sources, produced from the active ICRF antenna limiters by operating antennas in non-standard phasing, at various levels of low ICRH power [24]. These additional discharges have also allowed us to ensure that the different source levels do not affect the core $\mathrm{W}$ transport analysis, which is the main goal of this study. A summary of the results obtained in a series of 6 shots is presented in Fig. 5, where the ratio of the central density to a 


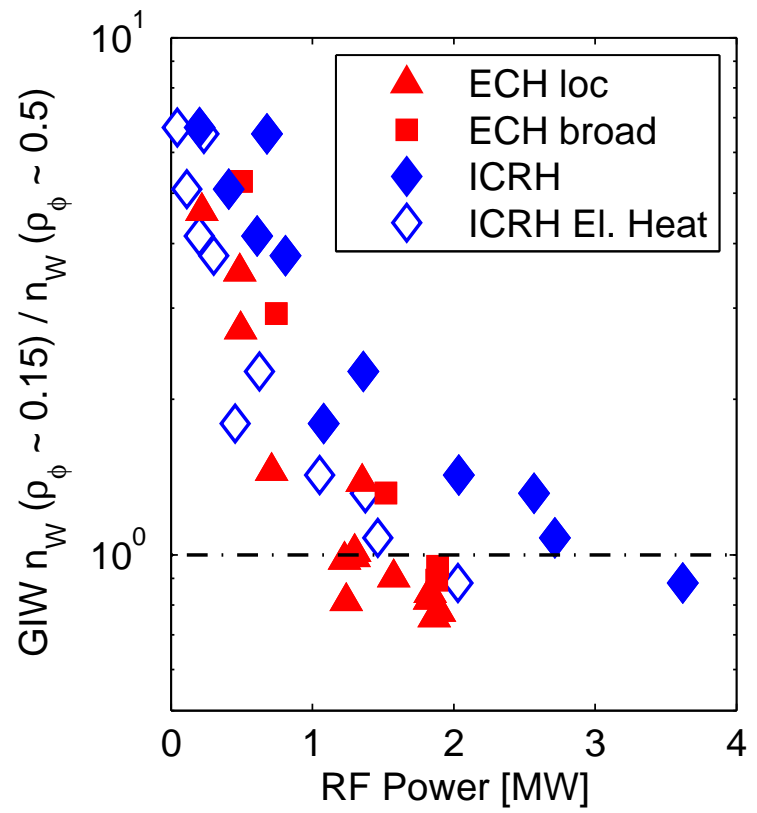

Figure 5. Ratio of central to peripheral W density measured by GIW as a function of the total RF heating power for phases with additional central localized ECRH (triangles), central broad ECRH (squares) and central ICRH (diamonds). The same ICRH phases are also represented in terms of the electron heating fraction of the ICRH power (open diamonds).

peripheral density of W (measured by GIW) and averaged over the different phases of constant heating power are plotted as a function of the total RF heating power. While this plot shows a certain level of scatter, it confirms the beneficial trend of increasing $\mathrm{RF}$ heating power to decrease the central $\mathrm{W}$ peaking. The most efficient effect is clearly obtained with localized ECRH, whereas broad ECRH deposition has a somewhat weaker effect, partly comparable to that of central ICRH, which however requires significantly higher total RF powers in order to obtain flat $\mathrm{W}$ density profiles of $\mathrm{W}$ in the central region. Since ICRH is delivering both electron and ion heating to the plasma, the same data points are plotted as a function of the ICRH electron heating power only (open diamonds in Fig. 5), as computed by the TORIC-SSFPQL package [46], and exhibit a trend very much consistent with that observed with ECRH. This result can be interpreted as an indication that, in these conditions, the $\mathrm{W}$ response is at least partly governed by the electron heating fraction produced by the additional RF heating. This consideration also suggests that an important element which determines the $\mathrm{W}$ density profile in the central region is related to the relative strength of the turbulent transport component with respect to the neoclassical transport component, consistent with previous studies [2]. Recent theoretical work has also pointed out that, at a fixed level of total turbulent heat flux, the turbulent transport of heavy impurities is maximum when the electron to ion heat flux ratio slightly exceeds unity [22]. This element will be taken into consideration in Section 3. In addition to the increase of the turbulent transport component with increasing $\mathrm{RF}$ heating, the $\mathrm{W}$ behavior is also determined 

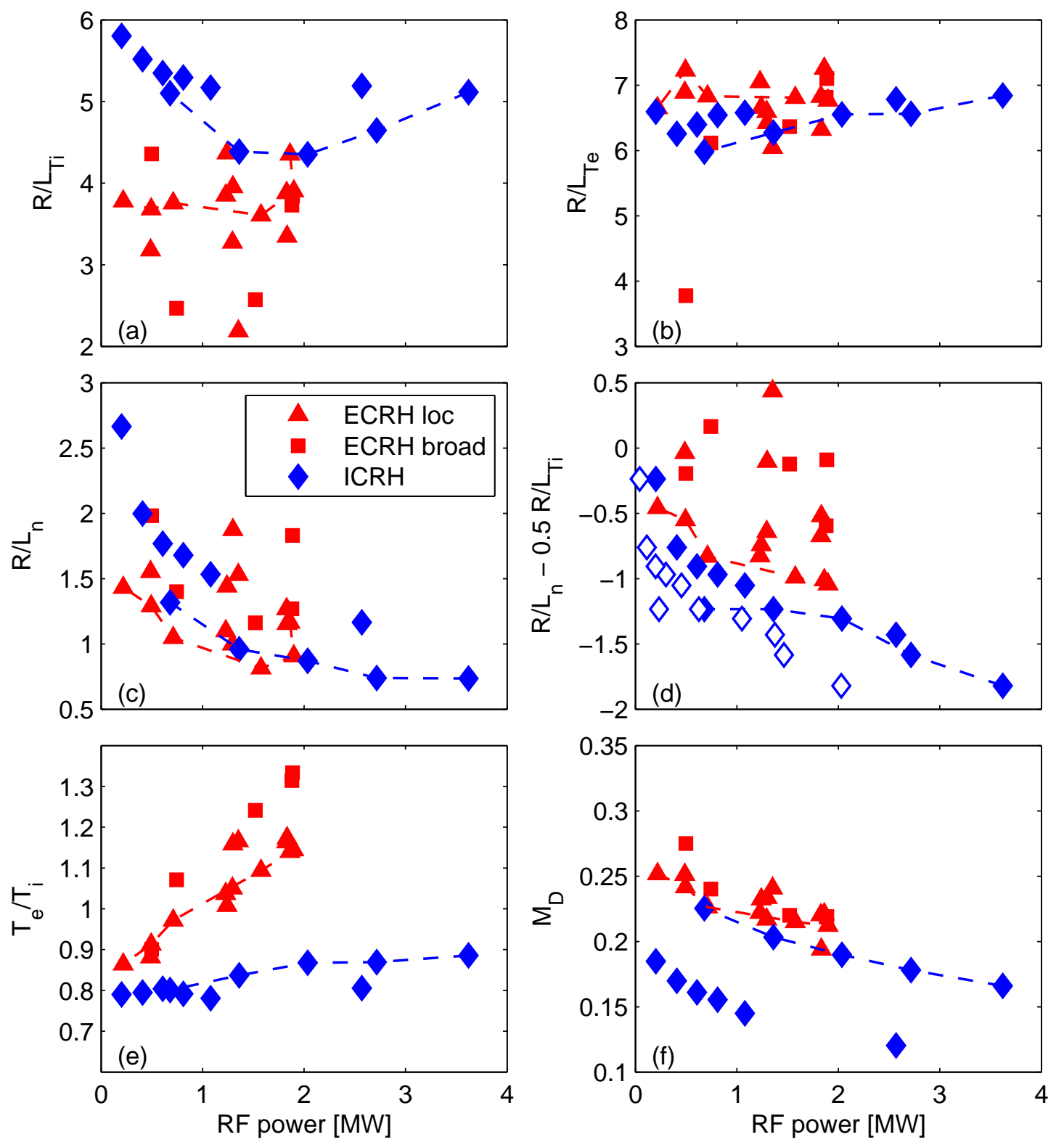

Figure 6. Normalized logarithmic ion temperature and electron temperature gradients $\left(R / L_{T i}\right.$ (a) and $R / L_{T e}$ (b) respectively) as well as normalized logarithmic density gradients $R / L_{n}$ (c) and simple neoclassical impurity peaking parameter $R / L_{n}-$ $0.5 R / L_{T i}$ (d), electron to ion temperature ratio $T_{e} / T_{i}(\mathrm{e})$ and thermal Mach number of Deuterium $M_{D}=v_{\phi} / v_{t h D}$ (f) averaged in the radial window $0.1 \leq r / a \leq 0.4$ and plotted as a function of the RF heating power in MW. Dashed lines identify the trajectories of discharges $\sharp 32404$ and $\sharp 32408$ presented in Figs. 1 and 2 respectively. 


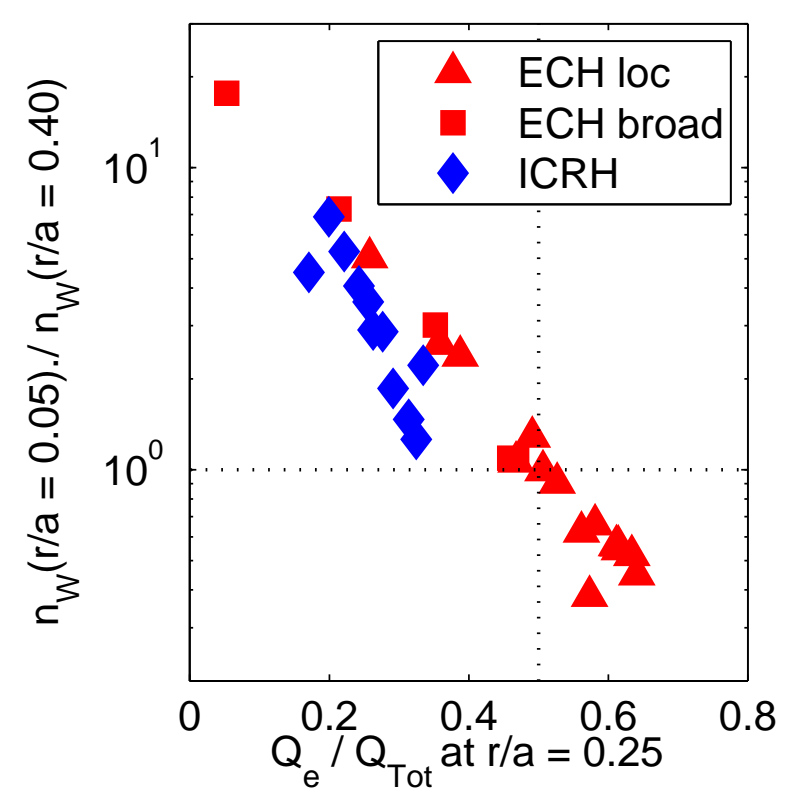

Figure 7. Peaking factor of the $\mathrm{W}$ density in the core as a function of the electron heat flux fraction inside $r / a=0.25$

by the change of the main plasma density, temperature and rotation profiles, which are also affected by the additional RF heating and on which the $\mathrm{W}$ neoclassical transport critically depends. Fig. 6 illustrates the dependece of the normalized logarithmic temperature and density gradients, as well as the electron to ion temperature ratio and the thermal deuterium Mach number averaged in the central region of the plasma $(r / a \leq 0.4)$ as a function of the RF heating power. We observe that plasmas with additional ICRH heating exhibit higher values of $R / L_{T i}$ and lower values of $T_{e} / T_{i}$ than cases with additional ECRH, consistent with an increased level of ion heating in the center produced by ICRH. Comparable values of $R / L_{T e}$ and equally decreasing dependences of the central Mach number are also observed (we notice that one ICRH shot has lower Mach numbers since, for technical reasons, it had to be performed with a different set of NBI sources, resulting in a reduced externally applied torque at the same NBI heating power). Also the electron density logarithmic gradient $R / L_{n}$ shows similar trends, which implies that plasmas with the highest ICRH powers achieve significantly lower values of the neoclassical pinch parameter $R / L_{n}-0.5 R / L_{T i}$ [25] as compared to the corresponding cases with highest ECRH power, which have the same amount of RF electron heating fraction. This is a direct positive consequence of the fact that ICRH also produces ion heating, an effect that can be expected to directly modify the neoclassical convection.

All of these considerations suggest to analyze the $\mathrm{W}$ behaviour in the core as a function of the fraction of the electron heating power absorbed in the central region of the plasma. This can be computed by means of interpretive power balance calculations, which have been performed with TRANSP [47], and which take into account the heating power density profiles of all the external sources, the Ohmic power, the radiated power as 
well as the equipartition power between electrons and ions. Fig. 7 shows that the ratio of central $(r / a=0.05)$ to the off-axis $(r / a=0.40)$ flux-surface-averaged W density is well ordered by the fraction of the total electron heating power absorbed inside that radial window $(r / a=0.25)$ (as computed with power balance analysis by TRANSP). Points are sampled by type of heating (localized ECRH, broad ECRH and ICRH). We observe that different types of heating produce comparable effects, with ICRH being slightly more efficient at the same fraction of the electron heat flux (of course, not in terms of total injected RF power). This can be regarded as the result of the more favorable neoclassical convection produced by the ion heating fraction of the ICRH. It is also interesting to observe that in these conditions the ratio $Q_{N B I, i} / Q_{N B I, e}$ of the ion to the electron heat flux provided by NBI only is above 3 at $r / a=0.25$, and decreases to 1.5 at the edge, that is, NBI is mainly producing ion heating in the central region of the plasma. Thereby, in these conditions, the role of both RF heating systems is to move from conditions of dominant ion heating to conditions of comparable electron and ion heating in the central region of the plasma.

In conclusion, the analysis of the experimental observations performed so far suggests that at least a part of the experimentally observed behavior of the $\mathrm{W}$ density is the result of the combination of two main effects. The first is the increase of turbulent W transport produced by the increase of the central heating and of the central electron heating fraction. The second effect is the modification of the neoclassical convection produced by the reduction of the central electron density gradient, which has a stronger effect for ICRH plasmas also due to the higher values of the ion temperature gradients. Finally, Figs. 5 and 7 also show that centrally hollow $\mathrm{W}$ density profiles are obtained with the highest fractions of electron heating, achieved by localized ECRH. In AUG, centrally hollow $\mathrm{W}$ density profiles are regularly observed in conditions of centrally localized ECRH in combination with the presence of saturated $(m, n)=(1,1)$ MHD modes [32,34] ( $m$ and $n$ are the poloidal and toroidal mode numbers respectively). Similar to the observations reported in [34], also in the heating phases examined in the present work, sawteeth are present and are characterized by the occurrence of $(1,1)$ modes, which appear early after the sawtooth crash and saturate over a large portion of the sawtooth period. The presence of these modes is an additional important element which can be expected to determine the shape of the $\mathrm{W}$ density profile in the most central region of the plasma.

\section{Modelling of the experimental results}

In this section the experimental results are examined with both simplified models and theoretical models in order to identify the main ingredients which are required to reproduce the experimental trends. In particular, the drift-kinetic code NEO [48-50] and the gyro-kinetic code GKW [51,52] are applied to compute the neoclassical and the turbulent $\mathrm{W}$ transport components using as inputs the measured profiles of the main plasma (deuterons and electrons).

A representative set of 12 heating phases from the experimental database presented 


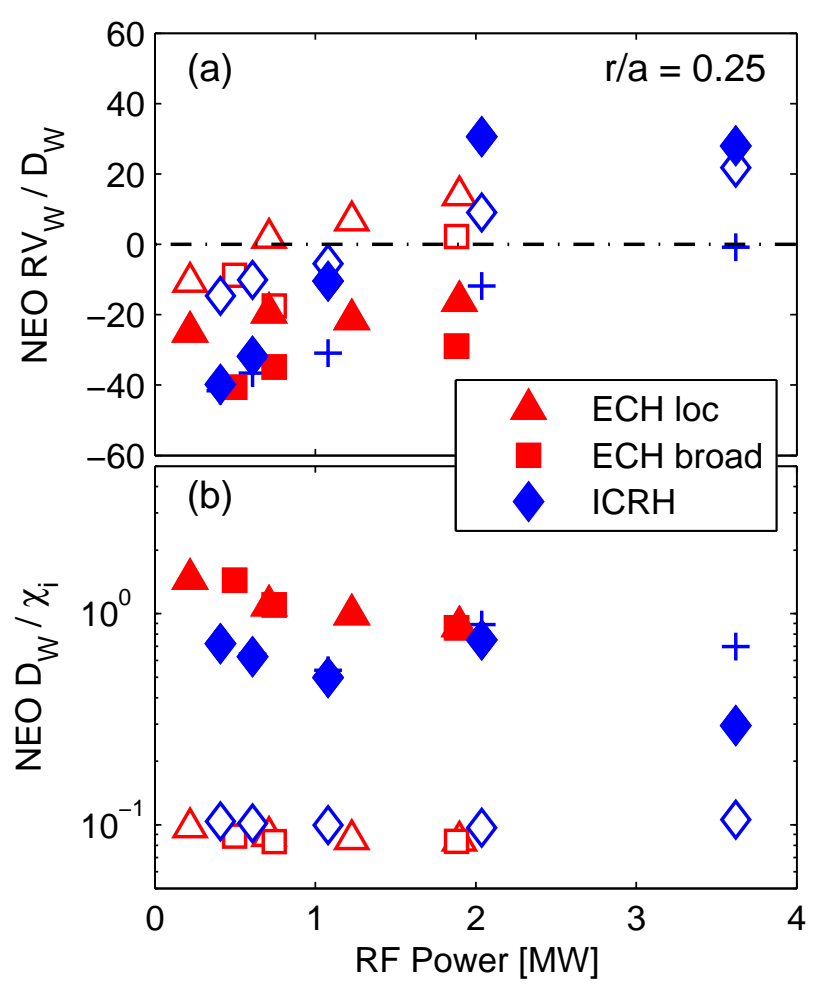

Figure 8. Transport coefficients at $\mathrm{r} / \mathrm{a}=0.25$ computed by NE0 with (full) and without (open) poloidal asymmetries of the $\mathrm{W}$ densities. Crosses ('+') show results with centrifugal effects only for the ICRH cases (impact of ICRH H minority is neglected).

in Section 2 is selected, out of which 5 are with auxiliary ICRH and 7 are with auxiliary ECRH.

Fig. 8 shows a comparison of the neoclassical results at $\mathrm{r} / \mathrm{a}=0.25$ obtained neglecting (open symbols) and including (full symbols) the poloidal asymmetries, as computed with the code NEO, and plotted as a function of the experimentally applied RF heating power. Different symbols show different types of heating, as reported in the legend. For the ICRH cases, we also show results which include the poloidal asymmetry produced by centrifugal effects only, and neglect the impact of the $\mathrm{H}$ minority (crosses ' +'). The presence of $\mathrm{H}$ minority also modifies the poloidal asymmetry of the $\mathrm{W}$ density, due to $\mathrm{H}$ minority temperature anisotropy [56-58]. Parallel and perpendicular pressures of the minority ions computed by the TORIC-SSFPQL [46] package have been used, and a model for the impact of the temperature anysotropy based on bi-Maxwellian distribution function [58] has been assumed in the NEO code. We observe that the neoclassical ratio of the $\mathrm{W}$ diffusion coefficient to the ion heat conductivity $D_{W} / \chi_{i}$ (Fig. 8b) is strongly increased by the centrifugal effects, consistent with previous studies $[33,43,44]$. We also observe that the impact of $\mathrm{H}$ minority is non-negligible in the cases with highest ICRH power, leading to a reduction of the parameter $D_{W} / \chi_{i}$, as a consequence of the reduction of the W LFS localization [29,30]. The inclusion of the poloidal asymmetry of the $\mathrm{W}$ density also modifies the $\mathrm{W}$ convection (Fig. 8a), leading 

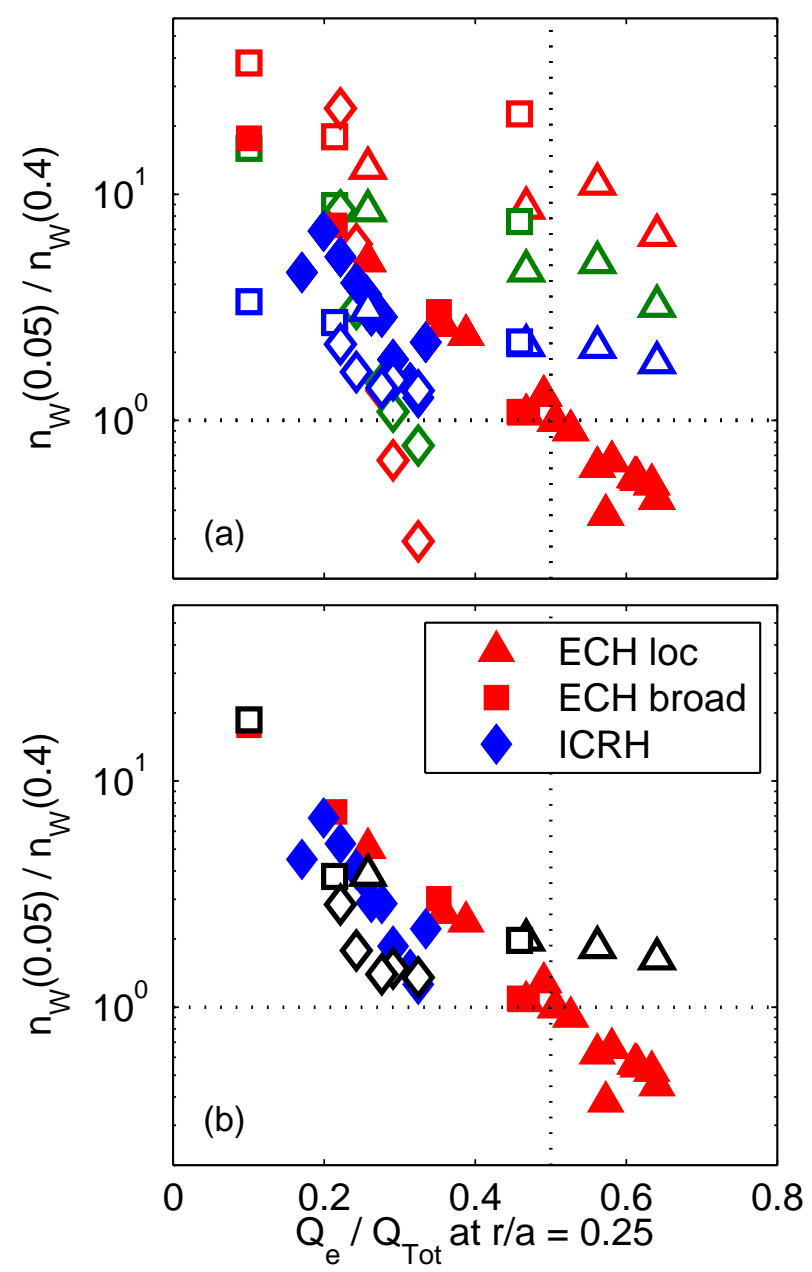

Figure 9. Experimental peaking factors of $\mathrm{W}$ density (already plotted in Fig. 7, full symbols) are compared to the results from the simple model Eq. 1 in open symbols, in (a) with constant $D_{\text {turb }} / \chi_{\text {eff }}=0$ (red), 0.1 (green), 1 (blue), in (b) with $D_{\text {turb }} / \chi_{\text {eff }}$ which fits the theoretical results of [22] as a function of $Q_{e} / Q_{i}$ (black)

to inward convection in the majority of cases, consistent with previous analyses $[29,44]$, The impact of the $\mathrm{H}$ minority leads to a reversal of the convection from inward (negative) to outward (positive) for the cases with highest ICRH power, due to an increase of the strength of the temperature screening resulting from a reduction of the LFS asymmetry of the $\mathrm{W}$ density produced by the centrifugal terms. This effect is also consistent with previous results $[29,43]$.

In order to investigate the impact of the $\mathrm{W}$ turbulent transport, as a first step, we introduce a simplified model for $\mathrm{W}$ transport in the central region of the plasma,

$$
\frac{R \Gamma_{W}}{n_{W}}=\left(D_{n e o}+\hat{D}_{\text {turb }} \chi_{\mathrm{eff}}\right) \frac{R}{L_{n W}}+R V_{\text {neo }},
$$

where the turbulent convection of $\mathrm{W}$ is neglected with respect to the neoclassical convection and where the factor $\hat{D}_{\text {turb }}$ stands as parameter for the ratio $D_{\text {turb }} / \chi_{\text {eff }}$. We use the effective conductivity $\chi_{\mathrm{eff}}=\left(Q_{i}+Q_{e}\right) /\left(n_{i} d T_{i} / d r+n_{e} d T_{e} / d r\right)$, which includes the impact of both ion and electron heating, and we notice that $\chi_{\mathrm{eff}}=\left(\chi_{i}+\chi_{e}\right) / 2$ when 

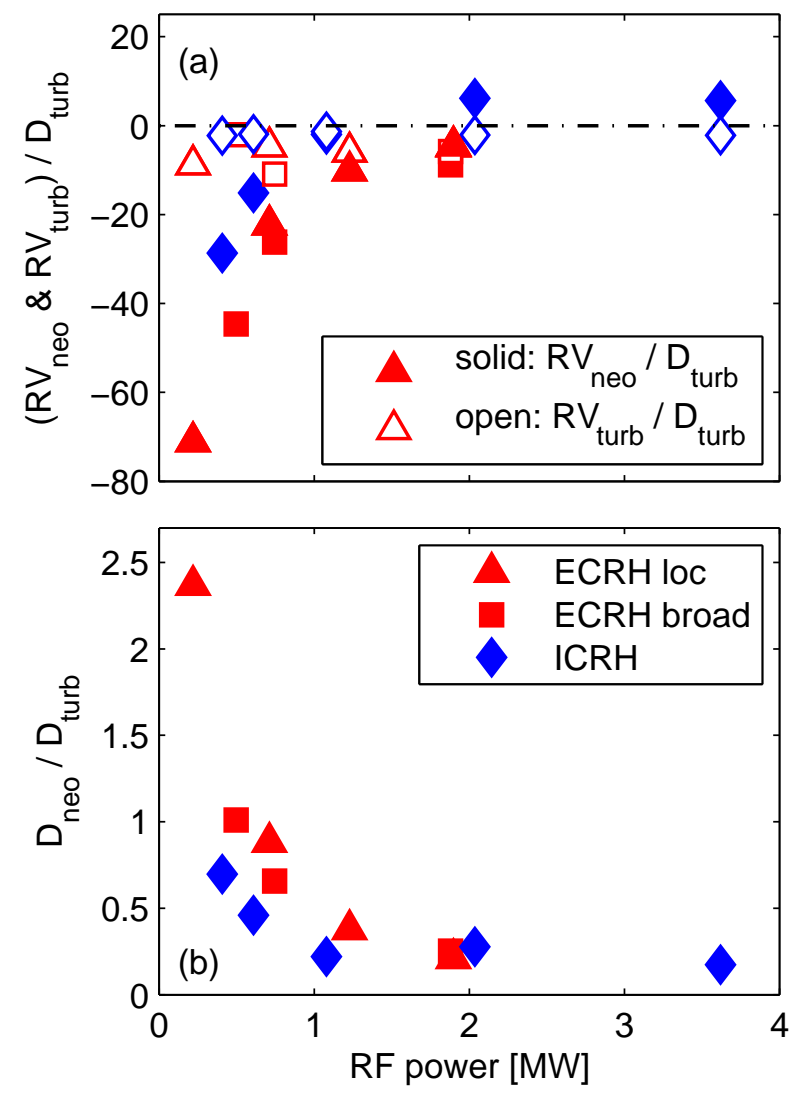

Figure 10. Predicted ratios of the neoclassical and turbulent convection to the turbulent diffusion (a) and ratio of the neoclassical diffusion to the turbulent diffusion (b) in the radial window $0.2 \leq r / a \leq 0.3$ as a function of the experimental additional RF heating power in MW. Neoclassical and turbulent transport coefficients have been computed by NEO and GKW respectively, and turbulent transport levels have been determined by assuming that the GKW effective heat conductivity matches the power balance anomalous effective heat conductivity computed by TRANSP.

$T_{i}=T_{e}$. In the framework of this model, using the neoclassical results of NEO of Fig. 8, we test whether a constant ratio of $D_{\text {turb }} / \chi_{\text {eff }}$ delivers results which are consistent with the experimental observations. Fig. 9(a) demonstrates that no choice of a constant $D_{\text {turb }} / \chi_{\text {eff }}$ is consistent with all of the data from low to high electron heat fraction. We also notice that neoclassical transport only (case with $D_{\text {turb }} / \chi_{\text {eff }}=0$ ) predicts too large peaking factors for most of the ECRH cases, whereas values of $D_{\text {turb }} / \chi_{\text {eff }} \geq 1$ are inconsistent with the observed accumulation at the lowest values of $Q_{e} / Q_{t o t}$. This suggests that only a variation of the turbulent ratio $D_{\text {turb }} / \chi_{\text {eff }}$ as a function of the electron heating fraction can explain the results, at least qualitatively in agreement with the theoretical results presented in [22]. By utilizing a polynomial fit of the theoretical results of $D_{\text {turb }} / \chi_{\text {eff }}$ as a function of $Q_{e} / Q_{i}[22]$, we obtain the results presented in Fig. 9(b), with a variation of the parameter $\hat{D}_{\text {turb }}$ from 0.08 to 4.1 from low to high values of $Q_{e} / Q_{\text {tot }}$ respectively. By introducing this variation of the ratio $D_{\text {turb }} / \chi_{\text {eff }}$, the resulting $\mathrm{W}$ peaking factors are more consistent with the experimental trend over the 

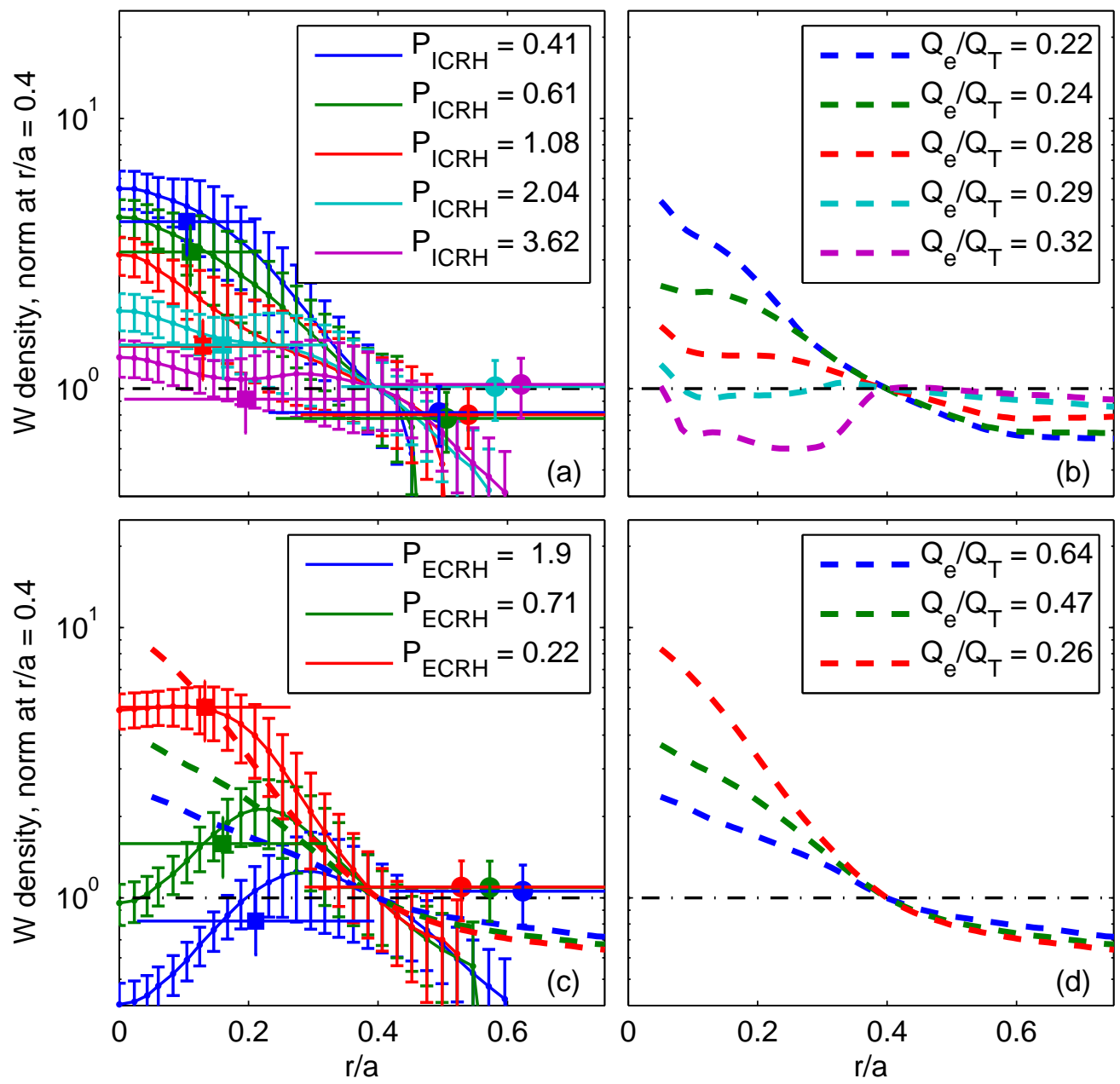

Figure 11. Measured (a,c) and predicted (b,d) flux-surface-averaged profiles.of W density, normalized at the correponding value at $\mathrm{r} / \mathrm{a}=0.4$, as a function of the normalized minor radius, obtained in RF power steps with $\operatorname{ICRH}(\mathrm{a}, \mathrm{b})$ and ECRH (c,d). In $(a, c)$ plot full squares and full circles are the related $\mathrm{W}$ density measurements of the GIW spectrometer (core and peripheral respectively). In the legends the corresponding values of the RF powers in MW (a,c) and the values of $Q_{e} / Q_{T}$ at $\mathrm{r} / \mathrm{a}=0.25(\mathrm{~b}, \mathrm{~d})$ are reported respectively. Predicted profiles have also been plotted with dashed lines in (c) to allow a more direct comparison with the experiment, solid lines with error bars.

entire experimental range of $Q_{e} / Q_{t o t}$, as compared to any assumed value of constant $D_{\text {turb }} / \chi_{\text {eff }}$.

The actual diffusive and convective components of turbulent transport have been computed by GKW linear runs at a single representative wave number $k_{y} \rho_{i}=0.4$, where the nonlinear spectra of $\mathrm{W}$ transport are usually found to peak in nonlinear gyrokinetic simulations [22]. The GKW calculations include the impact of poloidal asymmetries of the $\mathrm{W}$ density, also modified by the presence of the ICRH minority ions with the same model used in NEO. We combine turbulent and neoclassical transport components with the modelling approach described in $[33,43,44]$, and we normalize the turbulent transport components imposing that the predicted turbulent effective heat conductivity matches 


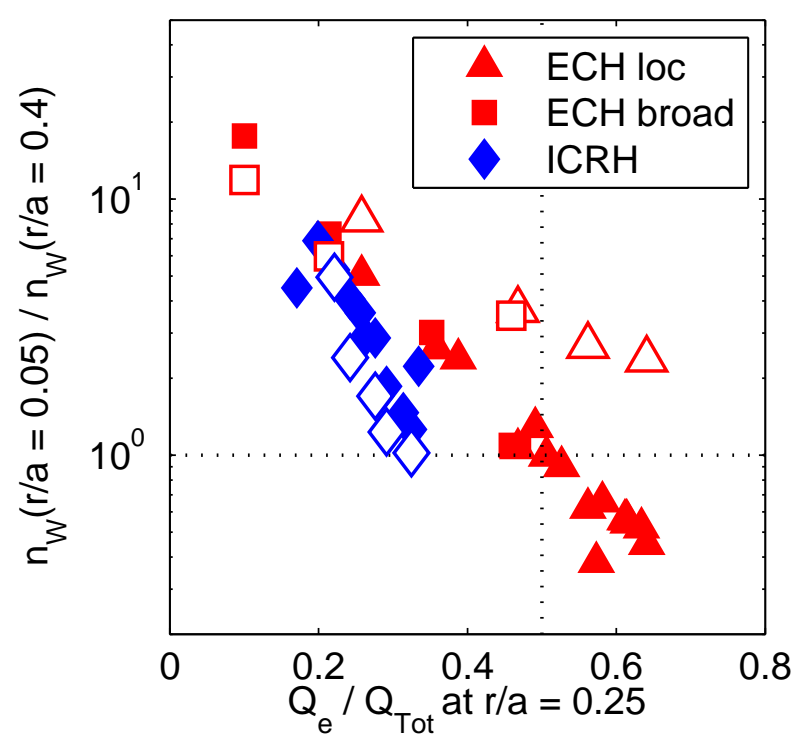

Figure 12. Same as Fig. 7, compared with the prediction of combined NEO and GKW linear calculations (open symbols).

the power balance anomalous effective heat conductivity $\chi_{\text {eff }}$, similarly to Eq. (1).

The relative impact of the predicted neoclassical and turbulent transport components in the radial window $0.2 \leq r / a \leq 0.3$ is presented in Fig. 10 as a function of the additional RF heating power. The turbulent convection (open symbols in Fig. 10(a)) is small as compared to the neoclassical convection (full symbols in Fig. 10(a)). A similar result is also obtained in a more external radial window $(0.35 \leq r / a \leq 0.45)$. At high additional RF heating powers, the neoclassical diffusion coefficient is smaller than the turbulent diffusion coefficient. The relative strength of the neoclassical diffusion coefficient increases in a more external radial window $(0.35 \leq r / a \leq 0.45)$, while exhibiting the same trends with increasing additional RF heating power.

The results of the combined NEO and GKW modelling are presented in Fig. 11, where predicted flux-surface-averaged density profiles are compared with those reconstructed from the experimental measurements, and in 12, where the predicted central peaking factor parameter is compared to the corresponding experimental results as a function of the fraction of electron heat flux.

The comparison shows that while some general trends are correctly reproduced by the modelling, complete quantitative agreement is not obtained. In particular, for the ECRH cases at high power, the central hollowness is not reproduced. The cases with ICRH are better reproduced by the modelling, where the inclusion of the impact of $\mathrm{H}$ minority ions also has a non-negligible effect on the jresults. However, the local hollowness of the predicted profile obtained with the highest ICRH power appears to be outside the experimental error bars and could result from an overprediction of the impact of the $\mathrm{H}$ minority asymmetry, as suggested by comparisons with 2D reconstructions of the $\mathrm{W}$ density distribution in this case.

The poor level of agreement of the ECRH cases with largest electron heat flux 
fraction reveals one of the critical limitations of the present modelling. While the variation of the local slope of the $\mathrm{W}$ density profile around $r / a=0.4$ is reasonably well reproduced (we note that experimental error bars become large outside $r / a=0.5$ due to the decrease of the electron temperature in relation to the sensitivity range of the SXR diagnostic), the central hollowness of the $\mathrm{W}$ profile is completely missed by the model. This result is not new and was somewhat expected, as a specific study on the interplay between central ECRH and saturated $(1,1)$ MHD activity has revealed [34]. This result also supports the hypothesis that the presence of the mode significantly impacts the $\mathrm{W}$ transport by means of a modification of the geometry of the confining magnetic field, which is assumed perfectly axisymmetric by the modelling activity presented in this work. Companion studies are taking into account the impact of the $3 \mathrm{D}$ modification of the magnetic equilibrium produced by a helical core [59] or a magnetic island [60]. The inclusion of these effects in the modelling with the NEO and GKW codes goes beyond the scope of the present paper, which is mainly dedicated to report the experimental results.

An additional problem is related to the difficulty of producing turbulent transport simulations which match the experimental electron to ion heat flux ratio in the innermost region and therefore produce the correct ratio of the $\mathrm{W}$ diffusion to heat conductivity. The level of disagreement on the electron to ion heat flux ratio which has been obtained by these single wave number linear gyrokinetic calculations with the nominal (measured) temperature and density profiles is shown in Fig. 13. Additional linear calculations with the GKW eigenvalue solver as well as nonlinear simulations are planned to investigate these aspects, in particular the potential role of subdominant modes in these experimental conditions, which can affect the nonlinear $\mathrm{W}$ turbulent transport [23]. We notice however that any modification of the turbulent convection should be large enough in comparison to the neoclassical convection, which appears unlikely to occur in these conditions where neoclassical convection is usually larger or comparable to the turbulent convection (Fig. 10). Finally, it is important to underline that ETG modes are also found unstable by GKW linear calculations at the nominal experimental parameters, and the actual fraction of electron heat flux which is produced at the large and intermediate scales which are relevant for $\mathrm{W}$ transport is difficult to assess quantitatively. This would require challenging multi-scale simulations in this central region of the plasma.

\section{Conclusions}

Experiments in AUG have been performed to directly compare the impact of central ECRH and central ICRH on the $\mathrm{W}$ behaviour. Decreasing steps of RF power have been applied to a constant background of NBI heating which robustly keeps the plasma in the H-mode confinement regime. The discharge conditions (high plasma current and low levels of gas puff) were such that in the absence of sufficient additional RF heating power $\mathrm{W}$ accumulates in the center, and in the presence of NBI only a sudden and uncontrollable accumulation takes place leading to the termination of the plasma discharge. These conditions favor the comparison between the two RF heating methods. 


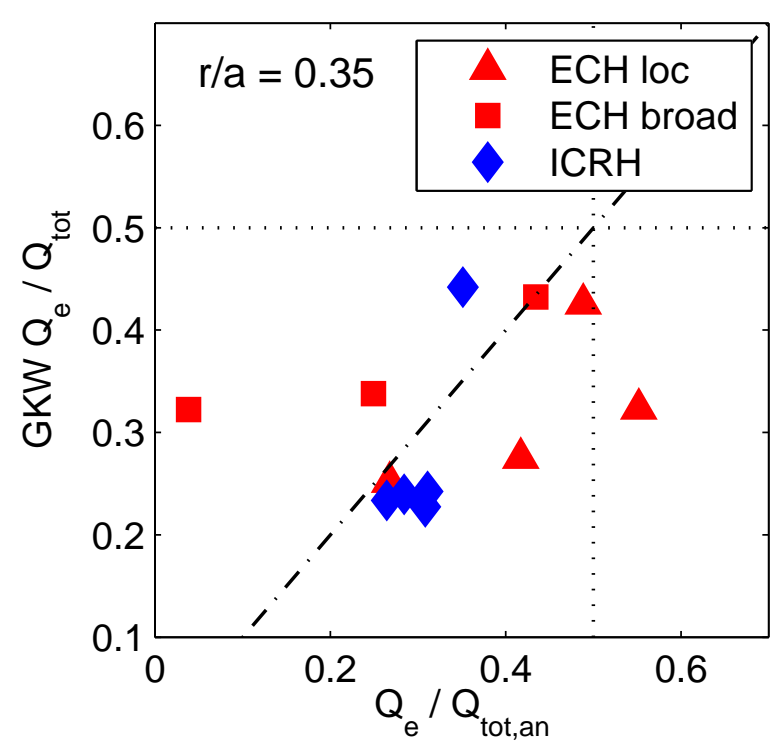

Figure 13. Fraction of the electron heat flux predicted by linear GKW calculations compared to TRANSP power balance

The most general observation is that ECRH and ICRH produce comparable effects on the central peaking of the $\mathrm{W}$ density when they produce comparable fractions of electron heating power. This also implies that in these conditions localized central ECRH is the most efficient method in terms of required total additional RF power in order to flatten the central $\mathrm{W}$ density profile. An interesting additional observation is that, at low levels of RF heating, stationary conditions are achieved in which the $\mathrm{W}$ density profile exhibits significantly stronger central peaking than the electron density, that is, W exhibits accumulation, but in stationary phases, without any consequent sudden and uncontrolled increase of the central W concentration. It has to be underlined that these stationary conditions with accumulation also exhibit regular sawteeth, therefore the experimental results alone do not allow us to ascertain how stationary these conditions of accumulation could be in the absence of regular sawteeth.

The analysis also reveals that the electron heating fraction is not the only parameter which governs the $\mathrm{W}$ response. Both central ECRH and central ICRH produce a beneficial reduction of the central peaking of the main plasma density profile. In addition, plasmas with additional central ICRH regularly exhibit stronger central ion temperature gradients as compared to ECRH plasmas, with consequent more favorable neoclassical convection. A combined modelling activity, which utilizes the drift-kinetic code NEO and the gyro-kinetic code GKW to compute the neoclassical and turbulent transport components, finds results with the same qualitative trends as the experiment, and provides evidences of quantitative agreement, particularly in the radial interval $0.3 \leq r / a \leq 0.5$. The modelling also shows that the impact of the temperature anisotropy of the $\mathrm{H}$ minority produced by ICRH has a non-negligible impact on the poloidal asymmetry of the $\mathrm{W}$ density, with favorable consequences on the neoclassical transport. The modelling reveals that the assumption of a constant ratio of the $\mathrm{W}$ 
diffusion to the heat conduction $D_{W} / \chi$ is not consistent with the observations, and supports the theoretical expectation that the dependence of $D_{W} / \chi$ on the electron to ion heat flux ratio is an important element in order to obtain the same trend as observed in these experiments. Finally, the modelling does not reproduce the local hollowness of the $\mathrm{W}$ density observed in the presence of the highest steps of ECRH power. This supports the hypothesis that the central hollowness is the consequence of the interplay between central ECRH and the observed $(m, n)=(1,1)$ MHD activity [34].

These experimental results confirm the applicability of both ECRH and ICRH for the suppression of $\mathrm{W}$ accumulation. There are however some considerations to be added in order to assess the relevance of these results for the extrapolation to plasma conditions of a reactor, and of ITER in particular. In these experiments both ECRH and ICRH have been applied in addition to a background of NBI heating, which is mainly delivering ion heating in the central region of the plasma. The absence of strong central NBI heating in an ITER plasma has some positive consequences with respect to the W behaviour. The negative impact of NBI, particularly connected with the production of a central particle source and with a strong toroidal torque, has been clearly identified in recent JET experiments [33]. In ITER, the small central fuelling from NBI allows limited central peaking of the plasma density. In addition, the much smaller ratio of torque to plasma inertia in ITER as compared to AUG or JET can be expected to lead to much smaller levels of toroidal rotation, with beneficial effects on the size and direction of the neoclassical transport. These beneficial conditions have been experimentally demonstrated in Alcator C-Mod in the absence of NBI heating [8]. In the central region of an ITER plasma (around $\rho \leq 0.3$ ) in the baseline scenario, the expected ratio of the electron to the ion heat flux strongly depends on the amount of auxiliary central electron heating which is applied, on the level of central radiated power and therefore of central W concentration, as well as on the transport model which is applied for the prediction [61-63]. In general, it can be expected to vary from a minimum of 0.5 to a maximum around 2 , a range which is covered by the variation explored in the experiments reported in this paper. Conditions with $Q_{e} / Q_{i} \simeq 1.5$ are theoretically predicted to benefit of the maximum increase of the turbulent diffusion of $\mathrm{W}$, while conditions with $Q_{e} / Q_{i} \ll 1$ and $Q_{e} / Q_{i} \gg 1$ are expected to be characterized by significantly lower values of the turbulent diffusion, for a given level of the total heat flux $\left(Q_{e}+Q_{i}\right)[22]$. While the experimental results presented in this paper can be considered to be at least qualitatively consistent with the prediction that the $\mathrm{W}$ diffusion increases with increasing ratio of $Q_{e} / Q_{i}$ starting from conditions of dominant ion heating, the equally important prediction that $\mathrm{W}$ diffusion decreases when $Q_{e} \gg Q_{i}$ still awaits an experimental validation. Since in present experiments conditions of $Q_{e} \gg Q_{i}$ are regularly achieved with small NBI heating, and therefore limited central fuelling, transient transport experiments dedicated to the separate measurement of diffusion and convection of the impurity could be more appropriate for this purpose (although likely more complex to carry out). In fact, it can be expected that, in the absence of central fuelling, the neoclassical transport is outward in the central region of the plasma, and therefore no central accumulation is obtained in those conditions, regardless the ratio of $Q_{e} / Q_{i}$, in 
contrast to the observations presented in this work. To this purpose, experiments with light impurities might be more appropriate with respect to heavy impurities, not only because they can be better diagnosed with charge exchange recombination spectroscopy, but also because their turbulent transport component largely dominates over the neoclassical transport component. Finally, as compared to plasmas in present tokamaks, in a tokamak plasma with size, current and field of a reactor, neoclassical transport is expected to play a more limited role [23], with the beneficial consequence of a significantly reduced risk of heavy impurity accumulation, as confirmed by recent transport modelling of ITER scenarios [63].

Acknowledgments C.A. acknowledges fruitful discussions with F. J. Casson, P. Mantica, A. Polevoi and M. Valisa. This work has been carried out within the framework of the EUROfusion Consortium and has received funding from the Euratom research and training programme 2014-2018 under grant agreement No 633053. The views and opinions expressed herein do not necessarily reflect those of the European Commission. ITER is the Nuclear Facility INB no. 174. The views and opinions expressed herein do not necessarily reflect those of the ITER Organization.

[1] Rice J.E., Bonoli P.T., Marmar E.S., Wukitch S.J., Boivin R.L., Fiore C.L., Granetz R.S., Greenwald M.J., Hubbard A.E., Hughes J.W., Hutchinson I.H., Irby J.H., Lin Y., Mossessian D., Porkolab M., Schilling G., Snipes J.A. and Wolfe S.M. 2002 Nucl. Fusion 42510.

[2] Dux R., Neu R., Peeters A.G., Pereverzev G., Mück A., Ryter F., Stober J., Plasma Phys. Control. Fusion 45, 1815 (2003).

[3] Neu R. et al 2003 J. Nucl. Mater. 313116.

[4] Puiatti M.E. and Valisa M. et al 2006 Phys. Plasmas 13042501.

[5] Valisa M. et al 2011 Nucl. Fusion 51033002.

[6] Pütterich et al 2013 Plasma Phys. Control. Fusion 55124036.

[7] Giroud C.et al 2015 Plasma Phys. Control. Fusion 57035004.

[8] Loarte A. et al 2015 et al Phys. Plasmas 22056117.

[9] Lerche E. et al 2016 Nucl. Fusion 56036022.

[10] Frojdh M., Liljestrom, Nordman H. 1992 Nucl. Fusion 32419.

[11] Angioni C. and Peeters A.G. 2006 Phys. Rev. Lett. 96095003.

[12] Dubuit N., Garbet X., Parisot T., Guirlet R., and Bourdelle C. 2007 Phys. Plasmas 14042301.

[13] Angioni C. et al 2007 Phys. Plasmas 14055905.

[14] Nordnam H et al 2008 Phys. Plasmas 15042316.

[15] Camenen Y., Peeters A.G., Angioni C. et al 2009 Phys. Plasmas 16012503.

[16] Fülöp T., Braun S., and Pusztai 2010 Phys. Plasmas 17062501.

[17] Fülöp T. and Moradi S. 2011 Phys. Plasmas 18030703.

[18] Howard N. T. et al 2012 Nucl. Fusion 52063002.

[19] Skyman A. 2012 Phys. Plasmas 19032313.

[20] Mollen A. et al 2012 Phys. Plasmas 19052307.

[21] Angioni C. et al 2012 Phys. Plasmas 19122311.

[22] Angioni C. et al 2015 Phys. Plasmas 22120501.

[23] Angioni C. et al 2017 Nucl. Fusion 57022009.

[24] Bobkov W. et al 2015 AIP Conf. Proceedings 1689030004.

[25] Hirshman S.P. and Sigmar D.J. 1981 Nucl. Fusion 211079.

[26] Wong S.K. 1987 Phys. Fluids 30818. 
[27] Romanelli M., Ottaviani M. 1998 Plasma Phys. Control. Fusion 401757.

[28] Fülöp T. and Helander P. 1999 Phys. Plasmas 63066.

[29] Angioni C, Helander P. 2014 Plasma Phys. Control. Fusion 56, 124001.

[30] Belli E., Candy J., Angioni C. 2014 Plasma Phys. Control. Fusion 56, 124002.

[31] Nave M. F. F. et al 2003 Nucl. Fusion 431204.

[32] Gude A. et al $201037^{\text {th }}$ EPS Conf. Plasma Physics, P4.124, ocs.ciemat.es/EPS2010ABS.

[33] Angioni C. et al 2014 Nucl. Fusion 54083028.

[34] Sertoli M. et al 2015 Nuclear Fusion 55113029.

[35] Hender T.C. et al 2016 Nucl. Fusion 56066002.

[36] Fussmann, G, Hofmann, J.V, Janeschitz G. et al 1989 J. Nucl. Mat. 162-164 14.

[37] Ida K., Fonck R.J, Sesnic S., Hulse R.A., LeBlanc B. and Paul S.F. 1989 Nucl. Fusion 29231.

[38] Fussmann G., Field A.R., Kallenbach A., Krieger K., Steuer K.-H. and the ASDEX Team 1991 Plasma Phys. Control. Fusion 331677.

[39] Perry M.E., Brooks N.H., Content D.A., Hulse R.A., Ali Mahdavi M. and Moos H.W. 1991 Nucl. Fusion 311859.

[40] Pasini D., Giannella R., Lauro Taroni L., Mattioli M., Denne-Hinnov B., Hawkes N., Magyar G. and Weisen H. 1992 Plasma Phys. Control. Fusion 34677.

[41] Rice J.E., Goetz J.A., Granetz R.S., Greenwald M.J., Hubbard A.E., Hutchinson I.H., Marmar E.S., Mossessian D., Sunn Pedersen T., Snipes J.A., Terry J.L. and Wolfe S.M. 2000 Phys. Plasmas 71825.

[42] Rice J.E. et al 2007 Fusion Sci. Technol. 51357.

[43] Casson F. J. et al 2015 Plasma Phys. Control. Fusion 57014031.

[44] Angioni C. et al 2015 Phys. Plasmas 22055902.

[45] Poli E., Peeters A.G. and Pereverzev G.V. 2001 Comp. Phys. Comm. 13690.

[46] Brambilla M. and Bilato R. 2009 Nucl. Fusion 49085004.

[47] Pankin A. et al 2004 Comput. Phys. Commun. 159157.

[48] Belli E. and Candy J. 2008 Plasma Phys. Control. Fusion 50095010.

[49] Belli E. and Candy J. 2009 Plasma Phys. Control. Fusion 51.

[50] Belli E. and Candy J. 2012 Plasma Phys. Control. Fusion 54015015.

[51] Peeters A.G. et al 2009 Comput. Phys. C. 1802650.

[52] Casson F. J. et al 2010 Phys. Plasmas 17102305.

[53] Bobkov V. et al 2016 Nucl. Fusion 56084001.

[54] Wagner, D., Stober, J., Leuterer, F. et al 2011 J. Infrared Milli Terahz Waves 32274.

[55] Sertoli M. et al 2015 Plasma Phys. Control. Fusion 57075004.

[56] Ingesson L. C. et al 2000 Plasma Phys. Control. Fusion 42161.

[57] Reinke M. L. et al 2012 Plasma Phys. Control. Fusion 54045004.

[58] Bilato R., Maj O. and Angioni C. 2014 Nucl. Fusion 54072003.

[59] Garcia-Regaña J. M. et al 2015 European Physical Society Conf. on Plasma Physics (Lisbon, Portugal) P2.170, http://ocs.ciemat.es/EPS2015PAP/html/author.html

[60] Bergman A. 2016 European Physical Society Conf. on Plasma Physics (Leuven, Belgium) P1.042, http://ocs.ciemat.es/EPS2016PAP/html/author.html

[61] Angioni C. et al 2009 Nucl. Fusion 49055013.

[62] Casper T. et al 2014 Nucl. Fusion 54013005.

[63] Loarte A. et al 2016 IAEA Fusion Energy Conference, Kyoto 2016, PPC/2-1. 